\title{
Quantum information processing in a spin-bus system of coupled chains
}

\author{
Xiang Hao and Shiqun Zhu* \\ School of Physical Science and Technology, Suzhou University, \\ Suzhou, Jiangsu 215006, People's Republic of China
}

\begin{abstract}
The effective Heisenberg interaction of long distance is constructed in spin qubits connected to a bus of two strongly coupled chains. Universal quantum computation can be realized on the basis of the bus which always keeps frozen at the ground state. It is found that the effective interaction is primarily determined by the energy spectra of the bus. With the variation of the distance between two connecting nodes, the interaction alternately occurs between antiferromagnetic and ferromagnetic ones. The long range interaction can also be attained in coupled infinite chains. The quantum gate operations with the high precision are implemented in the condition of quantum fluctuations.
\end{abstract}

PACS: 03.67.Lx,03.67.Pp, 03.65.Fd, 73.21.La

\footnotetext{
${ }^{*}$ Corresponding author; Electronic address: szhu@suda.edu.cn
} 


\section{INTRODUCTION}

The proposals for quantum information processing have important developments in the spin qubits of quantum dots [1, 2]. As the heart of solid state quantum computation, the forms of the exchange interaction between qubits have been recognized from the isotropic Heisenberg interaction [3, 4] to anisotropic ones [5]. The interactions have very short range in the context of these previous schemes. This often leads to constraints on the architecture [6] and serious technical obstacles [7]. Therefore, it is enlightening to find out one kind of long range effective interaction for quantum information processing. Recently, the scheme for a quantum bus of a spin chain has been put forward [8]. Through the electrical control of external spin qubits of Loss and DiVincenzo type [9], the long range isotropic Heisenberg interaction is demonstrated in the work [8] where the bus is considered as a effective qubit. However, after each quantum logic operation, the spin bus must be initialized into its working space. Apparently, this gives rise to the complexity of the operating mode. In condensed matters, coupled spin chains are very typical for quantum many body systems. Different

from one single spin- $\frac{1}{2}$ chain, two coupled chains exhibit the natural energy gap [10]. The gapped systems like two coupled spin chains can be experimentally prepared by the arrays of quantum dots. Here we show how to engineer two coupled chains as a spin bus for the realization of quantum information processing. Our method can identify arbitrary singlequbit gates and controlled-NOT one by electrically controlling the weak coupling between spin qubits and the bus. During the whole process, the spin bus cannot share quantum information, which reduces the complication in the operation sequence.

In this paper, two strongly coupled spin chains with the anisotropic Heisenberg XXZ exchange interaction are regarded as a bus. The form of effective interaction is analytically given in Sec. II. The effective interaction can be tuned by the variations of the anisotropy and weak coupling between spin qubits and the bus. In Sec. III, the universal set of quantum logic gates is realized. The effect of quantum fluctuations in the spin bus is taken into account. A discussion concludes the paper. 


\section{THE LONG RANGE EFFECTIVE INTERACTION}

In our method, the whole quantum system involves a bus of two coupled chains and external computational spin qubits which are weakly connected to the bus. For the qubits of the Loss and DiVincenzo type, the weak coupling can be electrically manipulated. If the bus system is always frozen at the ground state, the long range effective interaction can be attained at the computational qubits where quantum information can be efficiently processed. The total Hamiltonian in this system is written as

$$
H=H_{0}+H_{\text {in }}
$$

where $H_{0}$ is the Hamiltonian of the bus and $H_{\text {in }}$ describes the weak interaction between computational spin qubits and the bus. The general case of the bus system $H_{0}$ with the Heisenberg XXZ anisotropic exchange is considered as

$$
\begin{aligned}
H_{0}= & J \sum_{i=1}^{2} \sum_{j=1}^{L-1}\left(s_{i, j}^{x} s_{i, j+1}^{x}+s_{i, j}^{y} s_{i, j+1}^{y}+\Delta_{j} s_{i, j}^{z} s_{i, j+1}^{z}\right) \\
& +J \sum_{j=1}^{L} \vec{s}_{1, j} \cdot \vec{s}_{2, j}
\end{aligned}
$$

Here $\vec{s}_{i, j}$ denotes the spin operator at the $j$-th site of the $i$-th chain. The first item of Eq. (2) implies the chain with the anisotropy $\Delta_{j}=\Delta$ and second item describes the strong antiferromagnetic coupling between two chains. When the computational spin qubits $\vec{\tau}_{A}$ and $\vec{\tau}_{B}$ are weakly coupled to the bus at the connecting nodes $\vec{s}_{i, j}=\vec{s}_{m}$ and $\vec{s}_{i^{\prime}, j^{\prime}}=\vec{s}_{n}$, the interaction can be expressed by $H_{i n}=J_{A} \vec{\tau}_{A} \cdot \vec{s}_{m}+J_{B} \vec{\tau}_{B} \cdot \vec{s}_{n}$.

The bus system has the non-degenerate ground state $\left|\psi_{0}\right\rangle$ with the energy $\epsilon_{0}$ and the first excited state [10]. Due to so large energy gap, the system can be frozen at the ground state when the physical temperature $k T$ is much smaller than the gap. The effective Hamiltonian between two computational qubits $H_{e f f}^{(A, B)}$ can be obtained in this condition. Through the use of the standard canonical transformation formalism [11], the effective Hamiltonian of the whole system can be expressed by

$$
H_{e f f}=\left(\left\langle\psi_{0}|H| \psi_{0}\right\rangle+H_{e f f}^{(A, B)}\right) \otimes\left|\psi_{0}\right\rangle\left\langle\psi_{0}\right|
$$

Because the total spin at the ground state $\left|\psi_{0}\right\rangle$ is zero, the mean value $\left\langle\psi_{0}|H| \psi_{0}\right\rangle=\epsilon_{0}$. The effective Hamiltonian $H_{e f f}^{(A, B)}=-\sum_{k>0} \sum_{\lambda_{k}=0}^{d_{k}-1} \frac{\left\langle\psi_{0}\left|H_{i n} P_{k}^{\lambda_{k}} H_{i n}\right| \psi_{0}\right\rangle}{\epsilon_{k}-\epsilon_{0}}$. The projector $P_{k}^{\lambda_{k}}=$ 
$\left|\psi_{k}^{\lambda_{k}}\right\rangle\left\langle\psi_{k}^{\lambda_{k}}\right|$ where $\left|\psi_{k}^{\lambda_{k}}\right\rangle,\left(\lambda_{k}=0,1, \cdots, d_{k}-1\right)$ is the $k$-th excited degenerate state with the energy $\epsilon_{k}$ and $d_{k}$ is the degree of degeneracy for this state. Thus, the expression of $H_{e f f}^{(A, B)}$ is given by

$$
H_{e f f}^{(A, B)}=-\sum_{k, \lambda_{k}} 2 J_{A} J_{B} \sum_{\alpha} R e\left(m_{\alpha} n_{\alpha}^{*}\right) \tau_{a}^{\alpha} \tau_{b}^{\alpha}+C_{e f f}
$$

where the constant $C_{\text {eff }}=-\sum_{k, \lambda_{k}} \sum_{\alpha} \frac{J_{A}^{2}}{4}\left|m_{\alpha}\right|^{2}+\frac{J_{B}^{2}}{4}\left|n_{\alpha}\right|^{2}$. The parameters $m_{\alpha}=\frac{\left\langle\psi_{0}\left|s_{m}^{\alpha}\right| \psi_{k}^{\lambda_{k}}\right\rangle}{\sqrt{\epsilon_{k}-\epsilon_{0}}}$ and $n_{\beta}=\frac{\left\langle\psi_{0}\left|s_{n}^{\beta}\right| \psi_{k}^{\lambda_{k}}\right\rangle}{\sqrt{\epsilon_{k}-\epsilon_{0}}}$. It is shown that the parameters $m_{\alpha}\left(k, \lambda_{k}\right), n_{\beta}\left(k, \lambda_{k}\right)$ are determined by the energy spectra of the bus system. For this transient invariant quantum system $H_{0}$, the energy spectrum can be calculated. It is found out that these parameters satisfy the relation as

$$
-\sum_{k>0} \sum_{\lambda_{k}=0}^{d_{k}-1} m_{\alpha}\left(k, \lambda_{k}\right) n_{\beta}^{*}\left(k, \lambda_{k}\right)=\gamma_{m, n}^{\alpha} \delta_{\alpha, \beta}
$$

where $\gamma_{m, n}^{\alpha}$ is real and Eq. (5) is zero if $\alpha \neq \beta$. Moreover, $\gamma_{m, n}^{x}=\gamma_{m, n}^{y} \neq \gamma_{m, n}^{z}$ under the assumption of $\Delta \neq 1$. In general, the effective Hamiltonian of two computational qubits is written in form of the anisotropic Heisenberg interaction $H_{e f f}^{(A, B)}=2 J_{A} J_{B}\left[\gamma_{m, n}^{x}\left(\tau_{A}^{x} \tau_{B}^{x}+\tau_{A}^{y} \tau_{B}^{y}\right)+\right.$ $\left.\gamma_{m, n}^{z} \tau_{A}^{z} \tau_{B}^{z}\right]+C_{e f f}$. It is seen that the effective long range interactions $J_{m, n}^{\alpha}=2 J_{A} J_{B} \gamma_{m, n}^{\alpha}$ primarily rely on the properties of the bus.

To analytically give the effective interactions, the simplest case of $L=2$ is considered. The energy spectra and corresponding eigenstates of $H_{0}$ for $\Delta \in(0,1]$ are spanned in the product Hilbert space for all of the spins in the bus 


$$
\begin{aligned}
& \epsilon_{0}=J \eta_{0} \quad\left|\psi_{0}\right\rangle=a_{0}(|\downarrow \uparrow \downarrow \uparrow\rangle+|\uparrow \downarrow \uparrow \downarrow\rangle)+b_{0}(|\downarrow \downarrow \uparrow \uparrow\rangle+|\uparrow \uparrow \downarrow \downarrow\rangle)+c_{0}(|\downarrow \uparrow \uparrow \downarrow\rangle+\mid \uparrow \downarrow \downarrow \uparrow), \\
& \epsilon_{1}=-J \quad\left|\psi_{1}^{0}\right\rangle=\frac{1}{2}(|\downarrow \uparrow \uparrow \uparrow\rangle-|\uparrow \downarrow \uparrow \uparrow\rangle+|\uparrow \uparrow \downarrow \uparrow\rangle-|\uparrow \uparrow \uparrow \downarrow\rangle), \\
& \left|\psi_{1}^{1}\right\rangle=\frac{1}{2}(|\uparrow \downarrow \downarrow \downarrow\rangle-|\downarrow \uparrow \downarrow \downarrow\rangle+|\downarrow \downarrow \uparrow \downarrow\rangle-|\downarrow \downarrow \downarrow \uparrow\rangle), \\
& \epsilon_{2}=-\frac{J}{2}(1+\Delta) \quad\left|\psi_{2}\right\rangle=\frac{1}{\sqrt{2}}(|\downarrow \uparrow \downarrow \uparrow\rangle-|\uparrow \downarrow \uparrow \downarrow\rangle) \\
& \epsilon_{3}=-\frac{J}{2}(1-\Delta) \quad\left|\psi_{3}\right\rangle=\frac{1}{\sqrt{2}}(|\downarrow \downarrow \uparrow \uparrow\rangle-|\uparrow \uparrow \downarrow \downarrow\rangle) \\
& \epsilon_{4}=J \eta_{1} \quad\left|\psi_{4}\right\rangle=a_{1}(|\downarrow \uparrow \downarrow \uparrow\rangle+|\uparrow \downarrow \uparrow \downarrow\rangle)+b_{1}(|\downarrow \downarrow \uparrow \uparrow\rangle+|\uparrow \uparrow \downarrow \downarrow\rangle)+c_{1}(|\downarrow \uparrow \uparrow \downarrow\rangle+|\uparrow \downarrow \downarrow \uparrow\rangle), \\
& \epsilon_{5}=0 \quad\left|\psi_{5}^{0}\right\rangle=\frac{1}{2}(|\downarrow \uparrow \uparrow \uparrow\rangle+|\uparrow \downarrow \uparrow \uparrow\rangle-|\uparrow \uparrow \downarrow \uparrow\rangle-|\uparrow \uparrow \uparrow \downarrow\rangle), \\
& \left|\psi_{5}^{1}\right\rangle=\frac{1}{2}(|\downarrow \uparrow \uparrow \uparrow\rangle-|\uparrow \downarrow \uparrow \uparrow\rangle-|\uparrow \uparrow \downarrow \uparrow\rangle+|\uparrow \uparrow \uparrow \downarrow\rangle) \\
& \left|\psi_{5}^{2}\right\rangle=\frac{1}{2}(|\uparrow \downarrow \downarrow \downarrow\rangle+|\downarrow \uparrow \downarrow \downarrow\rangle-|\downarrow \downarrow \uparrow \downarrow\rangle-|\downarrow \downarrow \downarrow \uparrow\rangle), \\
& \left|\psi_{5}^{3}\right\rangle=\frac{1}{2}(|\uparrow \downarrow \downarrow \downarrow\rangle-|\downarrow \uparrow \downarrow \downarrow\rangle-|\downarrow \downarrow \uparrow \downarrow\rangle+|\downarrow \downarrow \downarrow \uparrow\rangle), \\
& \epsilon_{6}=\frac{J}{2}(1-\Delta) \quad\left|\psi_{6}\right\rangle=\frac{1}{\sqrt{2}}(|\downarrow \uparrow \uparrow \downarrow\rangle-|\uparrow \downarrow \downarrow \uparrow\rangle), \\
& \epsilon_{7}=\frac{J}{2}(1+\Delta) \quad\left|\psi_{7}^{0}\right\rangle=|\downarrow \downarrow \downarrow \downarrow\rangle, \\
& \left|\psi_{7}^{1}\right\rangle=|\uparrow \uparrow \uparrow \uparrow\rangle \\
& \epsilon_{8}=J \quad\left|\psi_{8}^{0}\right\rangle=\frac{1}{2}(|\downarrow \uparrow \uparrow \uparrow\rangle+|\uparrow \downarrow \uparrow \uparrow\rangle+|\uparrow \uparrow \downarrow \uparrow\rangle+|\uparrow \uparrow \uparrow \downarrow\rangle), \\
& \left|\psi_{8}^{1}\right\rangle=\frac{1}{2}(|\uparrow \downarrow \downarrow \downarrow\rangle+|\downarrow \uparrow \downarrow \downarrow\rangle+|\downarrow \downarrow \uparrow \downarrow\rangle+|\downarrow \downarrow \downarrow \uparrow\rangle), \\
& \epsilon_{9}=J \eta_{2} \quad\left|\psi_{9}\right\rangle=a_{2}(|\downarrow \uparrow \downarrow \uparrow\rangle+|\uparrow \downarrow \uparrow \downarrow\rangle)+b_{2}(|\downarrow \downarrow \uparrow \uparrow\rangle+|\uparrow \uparrow \downarrow \downarrow\rangle)+c_{2}(|\downarrow \uparrow \uparrow \downarrow\rangle+|\uparrow \downarrow \downarrow\rangle)
\end{aligned}
$$

Here the basis of the space $|\uparrow \downarrow \uparrow \downarrow\rangle$ labels $\left|\uparrow_{1,1}\right\rangle\left|\downarrow_{1,2}\right\rangle\left|\uparrow_{2,2}\right\rangle\left|\downarrow_{2,1}\right\rangle$ where $\left|\uparrow(\downarrow)_{i, j}\right\rangle$ is the eigenstate of the spin operator $s_{i, j}^{z}$ with the corresponding eigenvalues $\pm \frac{1}{2}$. The coefficients of Eq. (6) are given by $a_{f}=\frac{1}{\sqrt{2\left[1+1 /\left(\eta_{f}+\frac{1-\Delta}{2}\right)^{2}+1 /\left(\eta_{f}-\frac{1-\Delta}{2}\right)^{2}\right]}}, b_{f}=a_{f} /\left(\eta_{f}+\frac{1-\Delta}{2}\right), c_{f}=a_{f} /\left(\eta_{f}-\right.$ $\left.\frac{1-\Delta}{2}\right)$ and $\left\{\eta_{f=0,1,2} \mid \eta_{0}<\eta_{1}<\eta_{2}\right\}$ are the three real roots for the equation of $\eta^{3}+\frac{1+\Delta}{2} \eta^{2}-[2+$ $\left.\frac{(1-\Delta)^{2}}{4}\right] \eta-\frac{(1-\Delta)^{2}(1+\Delta)}{8}=0$. For the special case of $\Delta=1$, the roots $\left\{\eta_{f=0,1,2}\right\}=\{-2,0,1\}$ and the corresponding coefficients are obtained as $a_{0}=\frac{1}{\sqrt{3}}, b_{0}=c_{0}=-\frac{1}{2 \sqrt{3}}, a_{1}=0, b_{1}=c_{1}=-\frac{1}{2}$ and $a_{2}=b_{2}=c_{2}=\frac{1}{\sqrt{6}}$. Therefore, if the computational qubits are weakly coupled to the bus, we can calculate the effective interactions by $\gamma_{(1,1),(2,1)}^{\alpha}=\gamma_{1,2}^{\alpha}=\frac{1}{6 J}, \gamma_{(1,1),(2,2)}^{\alpha}=\gamma_{1,3}^{\alpha}=-\frac{1}{8 J}$ 
and $\gamma_{(1,1),(1,2)}^{\alpha}=\gamma_{1,4}^{\alpha}=\frac{1}{6 J}$. In the condition of $\gamma_{m, n}^{\alpha}<0$, the antiferromagnetic interactions $J_{m, n}^{\alpha}=2 J_{A} J_{B} \gamma_{m, n}^{\alpha}$ can be attained if the weak couplings are chosen as $J_{A} J_{B}<0$. The effects of the anisotropy on the long range interactions are shown by Fig. 1. It is seen that the effective interaction $\gamma_{1,2}^{x}$ is almost linearly decreased with the increase of the anisotropy $\Delta$ in Fig. 1(a). Here the effective anisotropy $\Delta_{1,2}=\gamma_{1,2}^{z} / \gamma_{1,2}^{x}$ is also illustrated by Fig. 1(b). These values almost take on the exponential increase. At the point of $\Delta=1$, the effective anisotropy is the maximal value of one where the isotropic Heisenberg interaction occurs. The connecting nodes of the bus are labeled in the new sequence given by Fig. $2(\mathrm{a})$. Similar to the results of [8], the effective interactions $J_{1, n}^{\alpha}$ alternately occurs between the antiferromagnetic and ferromagnetic ones. To clearly show this character, the effective interaction is plotted with respect to the distance $n-1$ between two nodes by Fig. 2 . It is found that the coefficient of the effective interaction $\gamma_{1, n}^{x}>0$ when the distance $n-1$ is odd. And the values of $\gamma_{1, n}^{x}$ decays almost exponentially. The opposite case is shown in the condition that $n-1$ is even. With the increase of the length of the chains, we also calculate the effective interactions. From Fig. 2(c), it is seen that $J_{1,2}^{x}$ is gradually increased to the certain value with length $L$. This fact demonstrates that the effective interaction can also be obtained in coupled chains with infinite size.

\section{THE REALIZATION OF QUANTUM LOGIC OPERATIONS}

After the evolution of the system, quantum information will be manipulated in the computational qubits under the assumption that the bus is always frozen at the ground state. It is the advantage that the distances of quantum logic operations can be enlarged much more. To implement the universal quantum computation, we need realize a set of quantum logic gates which consist of arbitrary single-qubit and controlled-NOT one. According to [5], an efficient way to construct the exact controlled-NOT logic gate can be provided for the general Heisenberg XXZ interaction

$$
U_{C N O T}^{(A, B)}=R_{B}^{y}\left(\frac{\pi}{2}\right) U_{C P F} R_{B}^{y}\left(\frac{\pi}{2}\right)^{\dagger}
$$

where the controlled phase flip gate $U_{C P F}=\exp \left[i \frac{2 \pi}{3} \overrightarrow{n_{1}} \cdot\left(\vec{\tau}_{A}+\vec{\tau}_{B}\right) / \hbar\right] \exp \left[i \frac{2 \pi}{3} \overrightarrow{n_{2}} \cdot\left(\vec{\tau}_{A}+\right.\right.$ $\left.\left.\vec{\tau}_{B}\right) / \hbar\right] U\left(\frac{\pi}{2}\right) R_{A}^{y}(-\pi) U\left(\frac{\pi}{2}\right) R_{A}^{x}\left(\frac{\pi}{2}\right) R_{B}^{x}\left(\frac{\pi}{2}\right)$. Here $R_{i}^{\alpha}(\theta)=\exp \left(-i \theta \tau_{i}^{\alpha} / \hbar\right)$ is the single-qubit rotation at the computational qubit $i=A(B)$. The unit directions are given by $\vec{n}_{1}=$ 
$\frac{1}{\sqrt{3}}\left(\vec{e}_{x}-\vec{e}_{y}+\vec{e}_{z}\right), \vec{n}_{2}=\frac{1}{\sqrt{3}}\left(\vec{e}_{x}+\vec{e}_{y}-\vec{e}_{z}\right)$. And the unitary operation $U\left(\frac{\pi}{2}\right)=\exp \left[\frac{-i}{\hbar} \int_{0}^{t_{c}} H_{e f f}^{(A, B)} d t\right]$ where $\frac{\pi}{2}=\int_{0}^{t_{c}} J_{m, n}^{x} d t / \hbar$. For the case of $\Delta=1$, the exact swap gate is obtained by $U_{\text {swap }}=\exp \left[\frac{-i}{\hbar} \int_{0}^{t_{s}} H_{\text {eff }}^{(A, B)} d t\right]$ and the swap time $t_{s}=\pi \hbar / J_{m, n}^{\alpha}$. From Eq. (7), it is seen that the controlled-NOT gate needs to be implemented by means of some single-qubit operations.

It is necessary to study how to operate the single-qubit rotations. When only one computational qubit $\vec{\tau}_{A}$ is coupled to the node $\vec{s}_{m}$ of the bus and the local magnetic field $\vec{b}$ is applied, the hamiltonian of the whole system is written by $H=H_{0}+\vec{b} \cdot \vec{\tau}_{A}$. Similarly, the effective hamiltonian can also be given by

$$
H_{e f f}=\left(\epsilon_{0}+\vec{b} \cdot \vec{\tau}_{A}+\frac{J_{A}}{4} \sum_{\alpha=x, y, z} \gamma_{m, m}^{\alpha}\right) \otimes\left|\psi_{0}\right\rangle\left\langle\psi_{0}\right|
$$

If the local magnetic field $\vec{b}=b_{\alpha} \vec{e}_{\alpha}(\alpha=x, y, z)$ is chosen, the unitary operation after the certain time is expressed by

$$
u(\theta)=\exp \left(-\frac{i}{\hbar} \int H_{e f f} d t\right)=R_{A}^{\alpha}(\theta) \cdot \exp \left(-\frac{i}{\hbar} \Phi\right)
$$

where $\theta=-\int b_{\alpha} d t, \Phi=\int\left(\epsilon_{0}+\frac{J_{A}}{4} \sum_{\alpha=x, y, z} \gamma_{m, m}^{\alpha}\right) d t$. It is shown that the single-qubit unitary rotation is operated by one computational qubit in the local field which is connected to the bus. Therefore, the universal quantum computation can be realized in the bus of two coupled chains.

It is noted that during the whole process of quantum logic operations the adiabatic criterion is satisfied in order to avoid the crossing of the energy levels. According to [8], the adiabatic criterion can be expressed by $t_{s}\left(\epsilon_{1}-\epsilon_{0}\right)>2 \pi \hbar$ for the case of $\Delta=1$. Owing to the previous work of [12], the energy gap of the bus $\epsilon_{1}-\epsilon_{0} \sim \frac{J}{2}+C J \exp \left(-\frac{L}{4}\right) / L$. From the above calculation, $t_{s}\left(\epsilon_{1}-\epsilon_{0}\right)>\frac{J \pi \hbar}{4 J_{A} J_{B} \gamma_{m, n}^{\alpha}}$. When the parameters $J_{A}=J_{B}=J / 10$, the value of $J / 4 J_{A} J_{B} \gamma_{m, n}^{\alpha}$ is about $10^{2}$ for coupled infinite chains. That is, the adiabatic criterion can be achieved $t_{s}\left(\epsilon_{1}-\epsilon_{0}\right)>100 \pi \hbar>2 \pi \hbar$. This shows that coupled chains with infinite size can be served as the bus for quantum information processing. If the actual physical parameters are considered as $J \simeq 1$ mev, $J_{A}=J_{B}=1 / 100, \Delta=1$, the operation time for two nodes $m=(1,1), n=(2,1)$ is $t_{c} \simeq 27$ ps.

In reality, the certain quantum fluctuations always exist in the bus. To clearly show the effects of the fluctuations on quantum computation, small variations of the anisotropy in the nodes of $\vec{s}_{m}, \vec{s}_{n}$ are considered by $\Delta_{m}=\Delta\left(1+\delta_{m}\right)$ and $\Delta_{n}=\Delta\left(1+\delta_{n}\right)$. It is found that the 
effective long range interaction can also be written in the form of Heisenberg XXZ exchange under this assumption. The norm of the operator $n=\left\|U\left(\delta_{m}, \delta_{n}\right)-U(0,0)\right\|$ is used to evaluate the error of the operators where $U\left(\delta_{m}, \delta_{n}\right)$ is the evolution operator of $H_{\text {eff }}^{(A, B)}$ after the time $t_{c}=\pi \hbar / 4 J_{A} J_{B} \gamma_{m, n}^{x}$ in the condition of the quantum fluctuations. And $U(0,0)$ corresponds to the one without the fluctuations. The error can be analytically given by

$$
n=\max \left\{\sqrt{2\left[1-\cos \frac{\pi \delta_{x}}{4}\right]}, \sqrt{2\left[1-\cos \frac{\pi \delta_{z}}{4}\right]}\right\}
$$

where the parameters $\delta_{x}=\frac{\gamma_{m, n}^{x}(0,0)-\gamma_{m, n}^{x}\left(\delta_{m}, \delta_{n}\right)}{\gamma_{m, n}^{x}(0,0)}$ and $\delta_{z}=\frac{\gamma_{m, n}^{z}(0,0)-\gamma_{m, n}^{z}\left(\delta_{m}, \delta_{n}\right)}{\gamma_{m, n}^{x}(0,0)}$. Here $\gamma_{m, n}^{\alpha}\left(\delta_{m}, \delta_{n}\right)$ is the coefficient of the effective interaction with the fluctuations of the anisotropy while $\gamma_{m, n}^{\alpha}(0,0)$ is the one without the fluctuations. The error of the operator is shown in Fig. 3. It is found that the error arrives at the minimum when $\delta_{m}=-\delta_{n}$. For a certain value of $\delta_{m}$, the error is increased with $\delta_{n}$. In the range of $\left|\delta_{m}\right|,\left|\delta_{n}\right|<0.005$, the order of the error can be decreased to $10^{-5}$ which is well below than the threshold $10^{-4}$ for the fault-tolerant quantum computation.

\section{DISCUSSION}

The gapped spin system of two coupled chains is served as the bus. During the whole process, the bus can always be frozen at the ground state for the large energy gap. By weakly connecting external computational spin qubits with the bus, the effective long range interactions can be attained. If two computational qubits are coupled, the effective hamiltonian has the form of anisotropic Heisenberg XXZ which can be useful for the construction of the controlled-NOT gate. It is found that the effective interaction alternately occurs between the antiferromagnetic and ferromagnetic ones. The effective interaction can be constructed in coupled chains with infinite size. The values decay almost exponential with increasing the distance of two nodes. In regard to only one qubit connected to the bus, single-qubit rotations can be implemented by the local magnetic field. Therefore, the universal quantum computation can be realized in the long range which can eliminate the technical difficulty arising from the exchange of short distances. Meanwhile, compared with the proposal of [8], the operating sequence of quantum information processing can be simplified. The quantum logic gates with small errors can be achieved in the condition of the quantum fluctuations of the anisotropy of coupled chains. 


\section{ACKNOWLEDGEMENT}

It is a pleasure to thank Yinsheng Ling, Jianxing Fang and Qing Jiang for their many fruitful discussions about the topic. The financial support from the special Research Fund for the Doctoral Program of Higher Education(Grant No. 20050285002) and the National Science Fund of China (Grand No. 10774108) are gratefully acknowledged.

[1] D. Loss and D. P. DiVincenzo, Phys. Rev. A 57, 120(1998).

[2] B. E. Kane, Nature(London), 393, 133(1998).

[3] X. Hu and S. Das Sarma, Phys. Rev. A 61, 062301(2000).

[4] G. Burkard, D. Loss and D. P. DiVincenzo, Phys. Rev. B 59, 2070(1999).

[5] F. Meier, J. Levy and D. Loss, Phys. Rev. Lett. 90, 047901(2003).

[6] D. A. Lidar and R. M. Noack, Phys. Rev. Lett. 88, 017905(2002).

[7] K. M. Svore, B. M. Terhal and D. P. DiVincenzo, Phys. Rev. A 72, 022317(2005).

[8] M. Friesen, A. Biswas, X. Hu, and D. Lidar, Phys. Rev. Lett. 98, 230503(2007).

[9] J. R. Petta, A. C. Johnson, J. M. Taylor, E. A. Laird, A. Yacoby, M. D. Lukin, C. M. Marcus, M. P. Hanson and A. C. Gossard, Science 309, 2180(2005).

[10] E. Dagotto and T. M. Rice, Science 271, 618(1996).

[11] A. Ferreira, J. M. B. Lopes dos Santos, arxiv: quant-ph/0708.0320v1(2007).

[12] S. R. White, Phys. Rev. Lett. 73, 886(1994). 


\section{Figure Captions}

Fig. 1

The effective interactions of $L=2$ are numerically plotted as a function of the anisotropy when the coupling $J=10, J_{A}=J_{B}=1$ (a). The coefficient of the effective interaction $\gamma_{1,2}^{x}$ is shown; (b). The effective anisotropy $\Delta_{1,2}$ is also illustrated.

Fig. 2

(a). The new sequence of the connecting nodes are labeled by the solid lines of the bus where the dash lines denote the couplings; (b). When one computational qubit is connected to the node $m=1$, the coefficient of the effective interaction $\gamma_{1, n}^{x}$ is plotted as the distance $n-1$ for $J=10, J_{A}=J_{B}=1, \Delta=0.2$ and $L=6$; (c). The effective interaction $J_{1,2}^{x}$ is plotted with increasing the length $L$ of chains for $J=10, J_{A}=J_{B}=1, \Delta=0.2$.

\section{Fig. 3}

The logarithm of the error $\log _{10}(n)$ in the chains with $L=4$ is plotted as a function of the anisotropy for $J=10, J_{A}=J_{B}=1, \Delta=0.2$ 

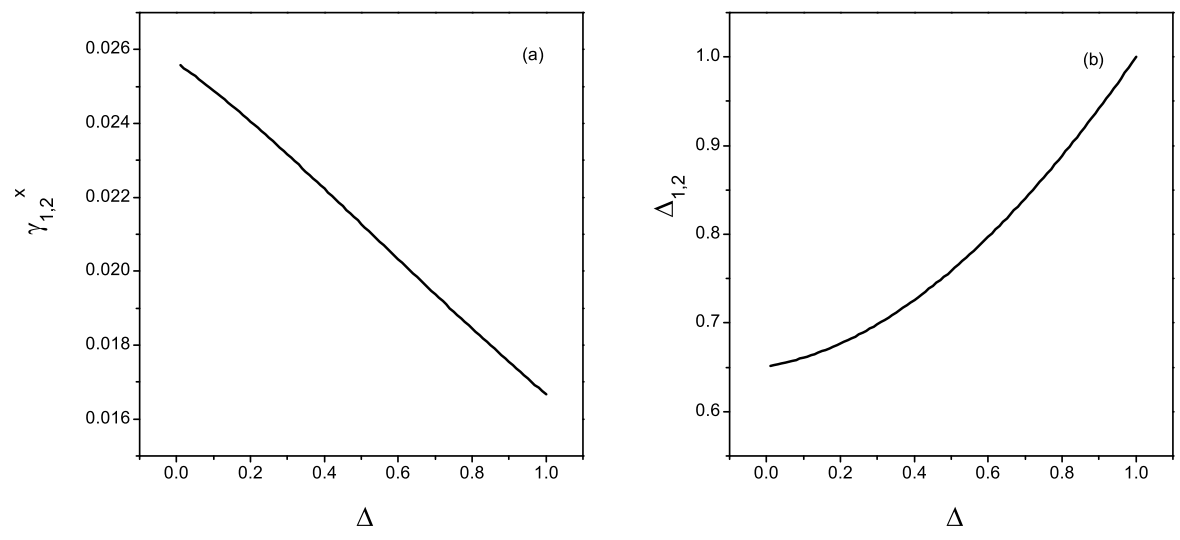

Fig. 1 


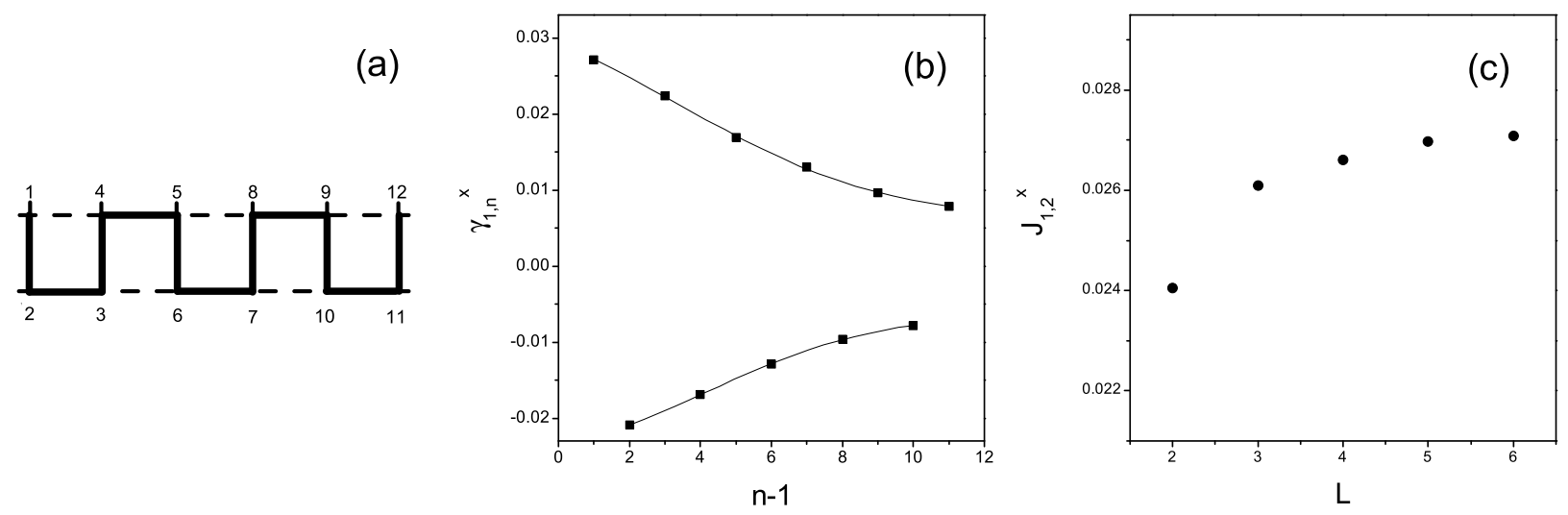


$\gamma$ 